\title{
Cavitation water treatment with GE USM cleaner device
}

\author{
Donka Stoeva ${ }^{1, *}$, Hafize Fidan $^{2}$, and Stanislava Tasheva ${ }^{3}$ \\ ${ }^{1}$ Department of Food Processing Machinery and Apparatuses, Technical Faculty, University of Food Technologies, Plovdiv, Bulgaria \\ 2 Department of Tourism and Culinary Management, Faculty of Economics, University of Food Technologies, Plovdiv, Bulgaria \\ ${ }^{3}$ Department of Heat Engineering, Technical Faculty, University of Food Technologies, Plovdiv, Bulgaria
}

\begin{abstract}
Cavitation treatment of surface and natural waters is a method for their microbiological purification. The aim of the present paper was to investigate the possibilities for the disinfection of natural waters by cavitation treatment with a Ge USM Cleaner device. A full factorial experiment of type 22 was performed. The factors influencing the water purification process were studied. Based on the obtained experimental results, the activation energy, the thermal effect due to the disinfection of the water and the rate constant of the process were calculated. The thermodynamic parameters of the transition state - Gibbs energy, enthalpy and entropy were also calculated. The analysis of the experimental results proveed the effectiveness of the method used for natural water treatment.
\end{abstract}

\section{Introduction}

The importance of water in the food industry, as in all other areas of life is undeniable. Due to the growing world's population and augmented food consumption, supplying clean water is becoming one of the most significant tasks of humanity. Surface and groundwater are subject to pretreatment (usually chemical) when used in the food industry. Wastewater from food enterprises is also treated by various chemical pretreatment methods before it is discharged back to the environment [1-3]. Regardless of the different goals of the water pretreatment and disinfection procedures, its main goal is the destruction of microorganisms. Due to the various unwanted side-effects of the chemical disinfection methods, the investigation for alternative methods with less unwanted effects is increasing. Attention is focused on the application of reagent-free water treatment technologies because they were considered as more ecologically friendly methods. The cavitation system is considered a promising method of wastewater treatment $[1,4]$. During cavitation, colloidal particles, which contain bacteria, are destroyed $[3,5]$. The destruction of microorganisms is the result of physical impact during cavitation. Under the conditions of hydrodynamic cavitation, the cell wall of microorganisms is broken. The bactericidal action of cavitation is directly related and proportional to its intensity and flow rate.

Formation of cavitation bubbles. The small bubbles are transported from a low-pressure area to areas of higher pressure. When the cavitation bubble disintegrates near a hard surface, a microinjection is formed (Fig. 1) [6]. Microcracks are formed in hard material, which causes great damage.

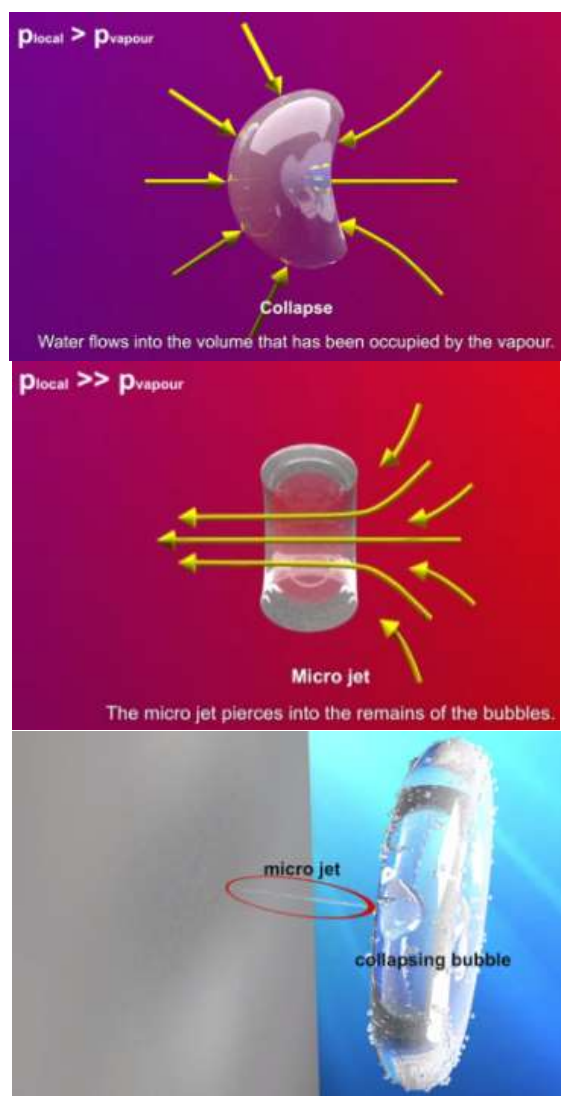

Fig. 1. Mechanism of bubble formation [6].

GE USM is the only device in which the fluid that passes through them in a hydrodynamic way, low and high-frequency mechanical oscillations, cavitation is generated and at the same time, a magnetic field is constantly exposed in space and variable during processing [7-8].

\footnotetext{
* Corresponding author: bodurova@gmail.com
} 
The device generates low-frequency and highfrequency oscillations, and at a certain degree of pressure cavitation and cavitation waves were generated in its vortex chamber. By processing with permanent magnets arranged in a certain way, a magnetic field is created in the device. As a result of all these effects described above, occurring simultaneously, the following changes of the liquid (water) were created [5-9]:

1) Declustering of molecules;

2) Reduction of surface tension;

3) A decrease in viscosity;

4) Biological decontamination;

5) Homogenization;

6) Preventing incrustations.

GE USM devices have the same principle of operation, have no moving parts, do not require a power supply and do not require maintenance [1]. The parameters by which the intensification of the process is achieved are hydrodynamic and geometric [7, 8]. To increase the degree of cavitation impact, the dimensionless ratio $\sigma$ is changed, which is defined as a cavitation number.

The aim of the present study was to investigate the influence of hydrodynamic cavitation on the microbiological treatment of river water from the Maritsa River, using the GE USM CLEANER device. A full factorial experiment of type $2^{2}$ for water treatment was made. Based on the experimental results, the activation energy, the rate constant of the process, the thermal effect due to disinfection, as well as the thermodynamic parameters (enthalpy, entropy, and Gibbs free energy) were determined.

\section{Material and methods}

A stand for cavitation water treatment [5, 9-11] and a device for generating cavitation GE USM were used in this study. Fig. 2 shows a section of the device and the passage of fluid through it. The device is a combination of low-frequency hydro-dynamic oscillations from 40 to $400 \mathrm{~Hz}$, ultrasonic oscillations over $20 \mathrm{kHz}$, cavitation waves and a constant magnetic field.

Collection of water samples from the sites: Water samples were collected in $10 \mathrm{~L}$ plastic bottles from Maritza River in the period from 01.05.2019 to 30.09 .2019 , transported in refrigerated boxes and stored at $4^{\circ} \mathrm{C}$ for a maximum $24 \mathrm{~h}$ before analysis. A total of nine samples were collected. The water samples were processed immediately for microbiological analysis.

Isolation and detection of the total count: Petri plates of Plate Count agar were used to determine the viable bacterial count. Each sample $(0.5 \mathrm{~mL})$ was transferred to the individual plates for the determination of total viable counts (TVC). The water samples $(0.1 \mathrm{ml})$ were preenriched in Luria Bertani broth and were serially diluted. The dilutions of 10-1 and 10-2 were used for plating and the plates were incubated at $37^{\circ} \mathrm{C}$ for $24 \mathrm{~h}$ [12].

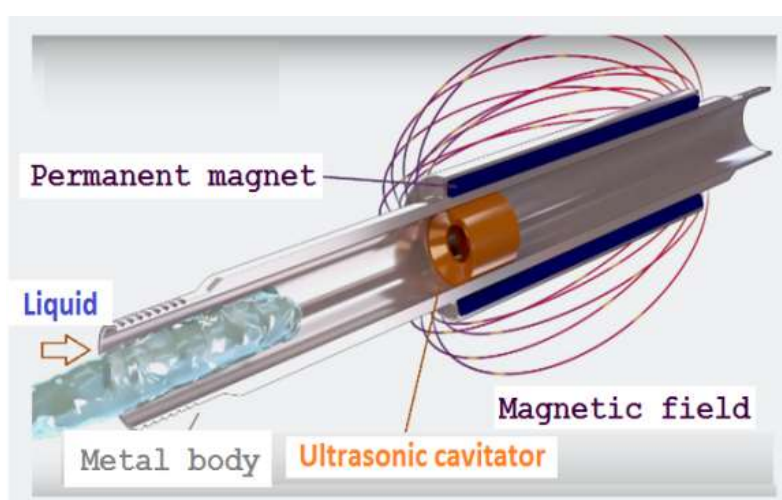

Fig. 2. Ge USM Cleaner device [15].

The total number of colonies on each plate was counted with the help of a colony counter and Colony Forming Unit (CFU) was counted using the formula:

$\mathrm{CFU}=$ No. of colonies $\mathrm{x}$ Dilution factor $/$ sample volume $(\mathrm{mL})$

Statistics: A factorial design ( $2^{2}$ type) was performed; the data were processed according to the reported number of destructed microorganisms in natural waters. The sequence of the experiment and the methodology for processing the results are prepared according to [13]. The mechanism of microbial cell destruction was previously described by [10, 14-16]. The cavitation erosion exerted the main influence on the level of microorganisms' destruction.

The coding of the factors and data for the values $2^{2}$ of $\mathrm{X}_{1}$ - time and $\mathrm{X}_{2}$ - cavitation number according [5, 10]; respectively upper, lower level: for $X_{1}$ is at 120 to $480 \mathrm{~s}$ and interval for variation is $180 \mathrm{~s}$; for $\mathrm{X}_{2}$ is at 0.172 to 0.34 and interval of variation 0.084 .

The cavitation number determines the intensity of cavitation; it is calculated based on the pressures in the cavitation zone (before and after).

Calculations: The thermal effect obtained during of the decontamination process of natural water is calculated. The following equation (1) presented in [17] was used for its calculation:

$$
Q_{t}=\mu_{t} / \mu_{0}
$$

where: $Q_{t}$ is the thermal effect resulting from the decontamination of natural water,

$\mu_{\mathrm{t}}-$ is the value of the experimentally obtained electrical conductivity of the water during in the experiment, $\mu \mathrm{S} / \mathrm{cm}$;

$\mu_{0}-$ is the standard value of the electrical conductivity of natural water $[18], \mu \mathrm{S} / \mathrm{cm}$;

$$
k=A e^{-E_{a} / R \cdot T}
$$

where: $\mathrm{A}$ is the Arrhenius constant, $\mathrm{R}$ is the universal gas constant, $T$ is the absolute temperature, $E_{a}$ is the activation energy.

$$
A=\frac{R \cdot T}{N \cdot h} e^{\Delta S^{\# / R}}
$$




$$
\begin{aligned}
& \Delta H^{\#}=E_{a}-R T, J / \mathrm{mol} \\
& \Delta G^{\#}=\Delta H^{\#}-T . \Delta S^{\#}, J / \mathrm{mol}
\end{aligned}
$$

where: $\mathrm{R}$ is the universal gas constant, $\mathrm{T}$ is the absolute temperature, $\mathrm{N}$ is Avogadro's constant, $\mathrm{h}$ is the Planck's constant, $\Delta \mathrm{S}^{\#}$ is the activation entropy, $\Delta \mathrm{H}^{\#}$ is the activation enthalpy, $\Delta \mathrm{G}^{\#}$ is the activation free energy or Gibb`s energy according the transition theory [20, 22-24].

\section{Results and discussion}

Table 1 presents the results of the $2^{2}$ factorial designs on the microbial count and electrical conductivity as response functions. The experiments were performed in triplicate. Mean values of the experimental results for the total microbial count and electrical conductivity during the process of natural water decontamination were given. Table 1. Full factorial experiment results for a total viable count and water conductivity.

Table 1. Full factorial experiment results for a total viable count and water conductivity.

\begin{tabular}{|c|c|c|c|c|c|}
\hline No & $\mathbf{X}_{\mathbf{0}}$ & $\mathbf{X}_{\mathbf{1}}$ & $\mathbf{X}_{\mathbf{2}}$ & $\begin{array}{c}\overline{\mathbf{Y}} \\
\text { Total mean viable } \\
\text { count, CFU/mL }\end{array}$ & $\begin{array}{c}\overline{\mathbf{Y}} \\
\text { Conductivity, } \\
\boldsymbol{\mu S} / \mathbf{c m}\end{array}$ \\
\hline $\mathbf{1}$ & + & - & - & 3100 & 318 \\
\hline $\mathbf{2}$ & + & - & + & 280 & 315 \\
\hline $\mathbf{3}$ & + & + & - & 220 & 315 \\
\hline $\mathbf{4}$ & + & + & + & 22 & 312 \\
\hline
\end{tabular}

A plan of the experiment has been prepared, the factors influencing the studied indicators have been determined based on previous studies [10]. Adequate regression equations for calculating the total viable count (6) and electrical conductivity (7) were derived, with correlation coefficient for equation $6\left(\mathrm{R}^{2}\right.$ is indicates that the model as fitted explains $56.25 \%$ ) and for equation $7\left(\mathrm{R}^{2}\right.$ is indicates that the model as fitted explains $\left.73.08 \%\right)$.

$$
\begin{aligned}
\bar{Y} & =905.5-784.5 . X_{1}-754.5 . X_{2} \\
\bar{Y} & =315.0-1.5 . X_{1}-1.5 . X_{2}
\end{aligned}
$$

Time and cavitation number are significantly effective in the experiment of both indicators - viable count and electrical conductivity. When the factors are at the upper level, the effect on the viability of the microorganisms is stronger. The presented regression equations substantiated that both factors (time and cavitation) influence equally the studied indicator.

The alteration in the number of destructed microorganisms depending on the combination of the factors obtained after processing the experimental data were shown in Fig. 3.

The graphical dependence shows that there were two effective factors on the total viable count in the treated water, and these were the duration of the experiment and the cavitation number. The relationship between the studied quantity and its affecting factors was linear, which is evident from the derived adequate regression model.

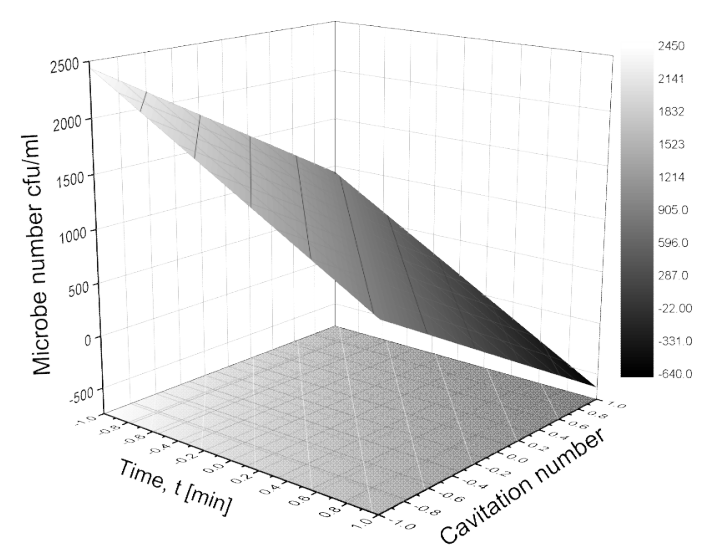

Fig. 3. Reflection surface and isolines for the alteration of the percentage of destructed microorganisms depending on the time and the cavitation number.

The increase in the duration of water treatment through the GE USM device led to increase and in cavitation number and decrease in the total viable number in the treated water sample.

The change in electrical conductivity depending on the combination of the individual significant factors obtained after processing the experimental data were shown in Fig. 4.

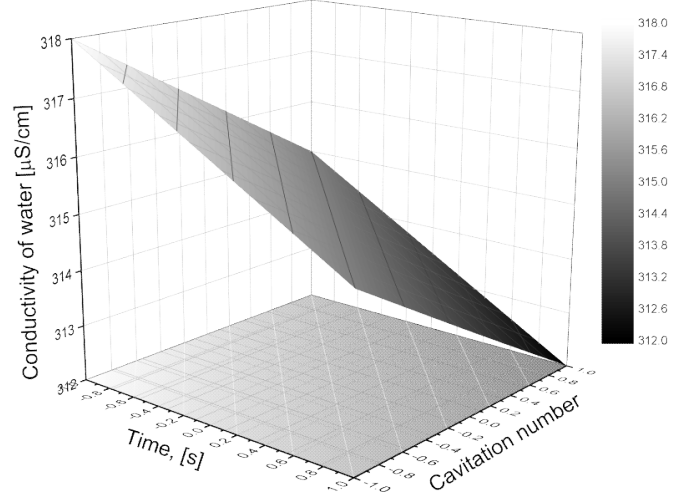

Fig. 4. Reflection surface and isolines for the alteration of electrical conductivity depending on time and cavitation number.

From the graphical dependence shown in Fig. 4, it can be seen that duration of the experiment and the cavitation number were the effective factors on the electrical conductivity of the treated river waters. The relationship between them was linear, as it also could be seen from the adequate regression model. It is known that as the temperature of the water increases, the electrical conductivity of the water decreases $[17,25]$. This will be due to an increase in the cavitation number of the stand and the duration of the passage of the treated water thought the GE USM device. Three independent studies were conducted for disinfection of natural waters. A graphical dependence reflecting the alteration of electrical conductivity depending on the duration of the experiment was constructed and presented in Fig. 5. A mathematical model calculated from the graphical dependence was 
shown in Fig. 5. It is represented by the following linear equation [17]:

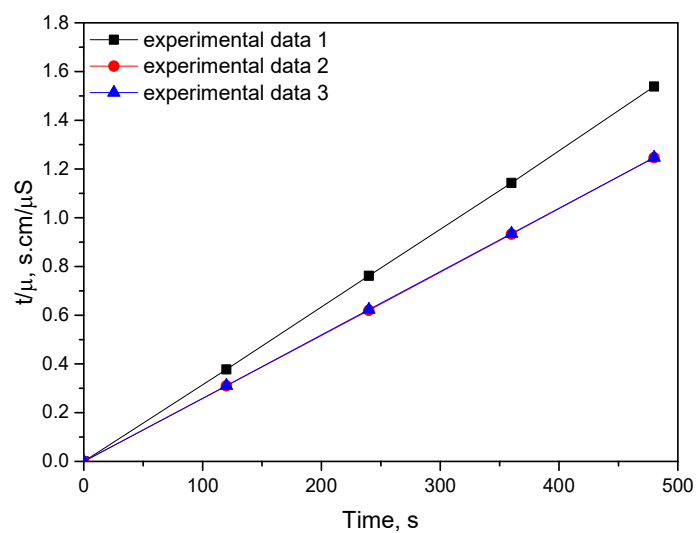

Fig. 5. Dependence of electrical conductivity as a function of time for decontamination of natural waters.

$$
\frac{t}{\mu}=\alpha+\beta . t
$$

where: $t$ is the duration of the experiment, $\mu$ is the alteration in electrical conductivity during the experiment, $\mu \mathrm{S} / \mathrm{cm}$, and $\alpha$ and $\beta$ are constants $[17,25]$. The obtained values of the model equation with real values, as well as the calculated speed constant of the process were presented in Table 2.

Table 2. The parameters of the values obtained from the linear equation.

\begin{tabular}{|c|c|c|c|c|}
\hline Experiment & $\boldsymbol{\alpha}, \mathbf{s . c m} / \boldsymbol{\mu S}$ & $\boldsymbol{\beta}, \mathbf{c m} / \boldsymbol{\mu} \mathbf{S}$ & $\mathbf{R}^{\mathbf{2}}$ & $\mathbf{k . 1 0}^{-\mathbf{3}} \mathbf{s}^{\mathbf{1}}$ \\
\hline $\mathbf{1}$ & 1.003 & 0.996 & 0.999 & 15 \\
\hline $\mathbf{2}$ & 1.003 & 0.999 & 1.000 & 5 \\
\hline $\mathbf{3}$ & 1.003 & 1.000 & 1.000 & 25 \\
\hline
\end{tabular}

The thermal effect obtained as the result of natural water treatment was calculated based on the experimental results from the full factorial design for the conductivity of the treated water. The results were given in Table 3 .

Table. 3 Calculated thermal effect for experimental results.

\begin{tabular}{|c|c|c|c|c|c|c|}
\hline \multirow{2}{*}{$\boldsymbol{\tau}, \mathbf{s}$} & \multicolumn{3}{|c|}{$\mathbf{T , K}$} & \multicolumn{3}{c|}{$\mathbf{Q}_{\mathbf{t}}$} \\
\cline { 2 - 7 } & $\mathbf{1}$ & $\mathbf{2}$ & $\mathbf{3}$ & $\mathbf{1}$ & $\mathbf{2}$ & $\mathbf{3}$ \\
\hline $\mathbf{0}$ & 293 & 295 & 297 & 0.184 & 0.243 & 0.242 \\
\hline $\mathbf{2}$ & 301 & 301 & 301 & 0.199 & 0.242 & 0.241 \\
\hline $\mathbf{4}$ & 305 & 305 & 307 & 0.197 & 0.242 & 0.241 \\
\hline $\mathbf{6}$ & 309 & 308 & 310 & 0.197 & 0.241 & 0.241 \\
\hline $\mathbf{8}$ & 313 & 312 & 313 & 0.195 & 0.241 & 0.241 \\
\hline
\end{tabular}

According to the values of the thermal effect obtained from the electrical conductivity data, the activation energy was determined according to Arrhenius' law, used also to calculate the thermodynamic parameters of the system enthalpy, entropy and Gibbs energy. The results were presented in Table 4. The negative values of activation energy and enthalpy reviled that the process flowed without bringing extra heat.
Table 4. Thermodynamic parameters of the experimental results.

\begin{tabular}{|c|c|c|c|}
\hline$\tau, S$ & $\begin{array}{c}\mathbf{E}_{\mathrm{a}}, \\
\mathrm{kJ} \cdot \mathrm{mol}^{-1}\end{array}$ & $\begin{array}{c}\Delta \mathbf{H}^{\#}, \\
\mathbf{k J} \cdot \mathrm{mol}^{-1}\end{array}$ & $\begin{array}{c}\Delta \mathbf{G}^{\#}, \\
\mathbf{k J}^{\prime} \cdot \mathbf{m o l}^{-1}\end{array}$ \\
\hline & \multicolumn{3}{|c|}{ Experiment 1} \\
\hline $\mathbf{0}$ & -2.95 & -5.38 & 66.33 \\
\hline 2 & -3.03 & -5.53 & 68.15 \\
\hline 4 & -3.07 & -5.60 & 69.05 \\
\hline 6 & -3.11 & -5.68 & 69.96 \\
\hline \multirow[t]{2}{*}{8} & -3.15 & -5.75 & 70.86 \\
\hline & \multicolumn{3}{|c|}{ Experiment 2} \\
\hline $\mathbf{0}$ & -3.13 & -5.58 & 66.63 \\
\hline 2 & -3.19 & -5.69 & 67.98 \\
\hline 4 & -3.23 & -5.77 & 68.89 \\
\hline 6 & -3.27 & -5.83 & 69.56 \\
\hline \multirow[t]{2}{*}{8} & -3.31 & -5.90 & 70.47 \\
\hline & \multicolumn{3}{|c|}{ Experiment 3} \\
\hline $\mathbf{0}$ & -3.14 & -5.61 & 67.01 \\
\hline 2 & -3.19 & -5.69 & 67.87 \\
\hline 4 & -3.25 & -5.80 & 69.28 \\
\hline 6 & -3.28 & -5.86 & 69.96 \\
\hline 8 & -3.31 & -5.92 & 76.61 \\
\hline
\end{tabular}

When calculating the activation entropy, the same value $\left(\Delta \mathrm{S}=-0.24 \mathrm{~kJ} \cdot \mathrm{mol}^{-1} \cdot \mathrm{K}^{-1}\right)$ was obtained for the three separate experiments. This shows that the system is thermodynamically stable and isolated. In addition, the environment was not considered as a factor that may influence the stability of the system. Gibbs energy as a parameter showed the irreversibility and spontaneity of the process; therefore a non-spontaneous process and an irreversible process occur from a thermodynamic point of view. The increase in the values of activating energy, enthalpy and Gibbs energy could be due to the temperature increase while the water passes through the cavitation stent and the device. According to the calculated thermal effect, the reason was the friction created while the water passes through the cavitation stand and the device.

\section{Conclusions}

The study has proved the effectiveness of the GE USM device in the cavitation method for water decontamination. The thermodynamic parameters (enthalpy, entropy and Gibbs energy) for the transition theory in this study revealed that the system was thermodynamically stable and not influenced by the environment and the conditions of the experiments. The process of natural waters' decontamination was spontaneous and irreversible, without requiring extra heat.

Acknowledgements: The authors thank Eng. Nikolai Arnaudski for the donated device Ge USM Cleaner. 


\section{References}

1. M. Sivakumar, S.Y. Tang, K.W. Tan, Ultrason. Sonochem. 21, 2069-2083 (2014)

2. N. Shopov, N. Vasilev, A. Bosakova-Ardenska. Proc. Ruse Uni. 56, 86-91 (2017)

3. A. Terziev I. Antonov, R. Velichkova, S. Antonov, Proc. Ruse Uni. 54, 124-126 (2015)

4. N. Krustev, P. Kostov, K. Atanasov, R. Petrova, Proceedings of the C\&SEE International Solid Waste Management Symposium (Institute of Thermodynamics and Energy Conversion, TUVienna, Vienna, 2009)

5. I. Antonov, M. Angelov, S. Antonov, Proceedings of the 13th Scientific Conference with International Participation TU-Sofia (EMF, Sozopol, 2008)

6. D. Bodurova, Cavitation treatment of liquid mediums, (PhD thesys, TU, Sofia, 2006).

7. M. Angelov, Proceedings of the 7th National Scientific Tech. Conf. Int. Participation Ecol. Health 2008 (Academic Publishing House of the Agrarian University, Plovdiv, 2008)

8. M. Angelov, Proceedings of the 17th Scientific Conference with International Participation TU-Sofia (EMF, Sozopol, 2012)

9. P.C. Sangave, A.B. Pandit, Ultrason. Sonochem. 11, 197-203 (2004)

10. D. Bodurova, M. Angelov, Proceedings of the International Conference: The Future of Food Safety Research: Contaminants and Influence of Agricultural Practices (FSRB, Brussels, 2004).

11. http://usm.tesbg.com/.

12. K R. Aneja, Experiments in Microbiology, Plant Pathology and Biotechnology. 4th edition (New Age International (P) Ltd, New Delhi, 2004)

13. A. Lambrem, Fundamentals of the engineering experiment in the study of machines and apparatus for the food industry (UFT, Plovdiv, 1994).

14. A. Pandit, K. Jyoti, Biochem. Eng. J. 14, 9-17 (2003)

15. A. Pandit, Biochem. Eng. J. 7, 201-212 (2001)

16. A. Pandit, Ultrason. Sonochem. J. 9, 123-131 (2002)

17. Q. Xu, Q. Shen, Colloids Surfaces B: Biointerf. 47, 98-101(2006)

18. Regulation No. 9/16 March 2001 on the quality of water intended for drinking and household purposes (Bulg. State Gazette, 30, 28 March 2001)

19. S. Sepidar, Z. Abidin, R. Yunus, A. Muhammad, Am. J. Appl. Sci. 6, 1390-1395 (2009)

20. H. Topallar, Ü. Geçgel, Turk J. Chem. 24, 247-253 (2000)

21. O. Levenspiel, Chemical reaction engineering (John Wily and Sons Inc., Hoboken, NJ, 2003).

22. A. Malijevsky, J. Novak, S. Labik, I. Malijevska, Physical chemistry in brief (Institute of Chemical Technology, Prague, 2005).
23. S. Tasheva, V. Gandova, V. Popova, T. Ivanova, Proceedings of the Youth Forums: Science, Technology, Innovation, Business - 2019 (House of Science and Technology, Plovdiv, 2019)

24. S. Tasheva, V. Gandova, S. Damianova, A. Stoyanova, J. Chem. Technol. Metall. 55, 45-51 (2020)

25. H. Wang, L. Chen, L-L. Weng, M-Y. Zhang, Q. Shen, J. Adh. Sci. Technol. 28, 2416-2423 (2014) 